\title{
- Dorothy Porter Wesley e a Organização do Conhecimento Negro na Coleção Especial Moorland- Spingarn Research Center
}

\author{
Dorothy Porter Wesley and the Black Knowledge \\ Organization in the Moorland-Spingarn Research Center \\ Special Collection
}

\author{
Franciéle Carneiro Garcês da Silva a,* (iD \\ Dirnéle Carneiro Garcez b (D) \\ Rodrigo de Sales c (it) \\ Gustavo Silva Saldanha d $\mathbb{D}$
}

\begin{abstract}
RESUMO: Este artigo apresenta a bibliotecária negra', Dorothy Porter Wesley, com especial enfoque em sua atuação na organização da Coleção Especial do Moorland-Spingarn Research Center. Com base em materiais bibliográficos e documentais, evidencia a denúncia realizada pela bibliotecária sobre a abordagem etnocêntrica, preconceituosa e limitante da Classificação Decimal de Dewey para catalogação dos recursos informacionais da Coleção. Para resolver essa lacuna, Porter Wesley preparou diversas bibliografias e catálogos destinados a organizar o conhecimento elaborado por e sobre as populações negras, africanas e da diáspora da Coleção. O estudo investiga o desenvolvimento crítico de sua trajetória e o papel metaepistemológico e metametodológico que conjuga a apropriação de ferramentas do campo informacional - a começar, a bibliografia -, como forma de luta, de resistência e de emancipação da comunidade negra americana. A pesquisa, em seus resultados, atesta a potencialidade revolucionária da práxis de Dorothy Porter Wesley, assim como fundamenta outra via epistemológico-histórica de concepção do campo biblioteconômico-informacional.
\end{abstract}

Palavras-chave: Organização do Conhecimento; Conhecimento Negro; Biblioteconomia Negra - Estados Unidos.

ABSTRACT: This article presents the black librarian, Dorothy Porter Wesley, with a special focus on her role in organizing the Special Collection of the Moorland-Spingarn Research Center. Based on bibliographic and documentary materials, we highlight the denunciation made by this black librarian about the ethnocentric, prejudiced and limiting approach of the Dewey Decimal Classification for cataloging the informational resources of the Collection. To address this gap, Porter Wesley has prepared several

\footnotetext{
a Programa de Pós-Graduação em Ciência da Informação, Escola de Ciência da Informação, Universidade Federal de Minas Gerais, Belo Horizonte, MG, Brasil.

b Programa de Pós-Graduação em Ciência da Informação, Universidade Federal de Santa Catarina, Florianópolis, SC, Brasil.

c Departamento de Ciência da Informação, Universidade Federal de Santa Catarina, Florianópolis, SC, Brasil.

d Instituto Brasileiro de Informação em Ciência e Tecnologia; Universidade Federal do Estado do Rio de Janeiro, Rio de Janeiro, RJ, Brasil.
}

* Correspondência para/Correspondence to: Franciéle Carneiro Garcês da Silva. E-mail: francigarces@yahoo.com.br.

Recebido em/Received: 30/06/2021; Aprovado em/Approved: 13/09/2021.

Artigo publicado em acesso aberto sob licença CC BY 4.0 Internacional ()(1)

${ }^{1}$ Não usaremos o termo "de cor" para se referir a pessoas negras americanas a não ser quando a passagem abordar uma citação da própria Dorothy Porter Wesley, embora seu uso popular 
bibliographies and catalogs designed to organize the knowledge produced by and about the Negro², African and diaspora populations of the Collection. The study investigates the critical development of her trajectory and the metaepistemological and metamethodological role that combines the appropriation of tools from the informational field - starting with bibliography - as a form of fight, resistance and emancipation for the American Negro community. As main results, this research attests to the revolutionary potentiality of the praxis of Dorothy Porter Wesley, as well as substantiating another epistemological-historical way of conception of the library and information science.

Keywords: Knowledge Organization; Black Knowledge; Black Librarianship - United States.

\section{INTRODUÇÃO}

No final do século XIX e início do século XX, diversos foram os movimentos e atores que se ativeram à busca por justiça social, oportunidades e lutas por direitos civis básicos dentro da sociedade americana. Assim como no Brasil, o projeto democrático dos Estados Unidos historicamente excluiu da formação e da produção de conhecimento as populações de origem africana, latina e caribenha, sobretudo dos espaços de produção de saber e intelectualidade e de representação de seus grupos étnico-raciais e geopolíticos em locais de poder (SILVA; SALDANHA, 2018, 2019; SILVA, 2019, 2020).

A presença do racismo, sexismo, preconceito, segregacionismo e diversas formas de opressões sofridas pela população afro-americana encontram-se registradas e denunciadas nas páginas de obras de bibliotecários e bibliotecárias negras como E. J. Josey, Virginia Lacey Jones, Clara Stanton Jones, Rebecca Hankins, Andrew P. Jackson, Dorothy Porter Wesley, entre outras e outros. No entanto, suas ações profissionais também foram decisivas para o desenvolvimento de movimentos civis e profissionais pelo direito à informação, à biblioteca e aos livros pelos cidadãos e cidadãs afroamericanas (JORDAN; JOSEY, 1977).

Durante a era segregacionista formal, juridicamente justificada, ou seja, perante o espaço-tempo da violência institucionalizada pela lei, as mulheres negras foram aquelas que "reagiram às políticas de exclusão generalizadas da sociedade branca, promovendo ideologias e estratégias de autoajuda, ao mesmo tempo que respondiam criativamente às sufocantes convenções de gênero dentro das comunidades negras" (DAGBOVIE, 2004, p. 241, tradução nossa). Através de suas lutas, trouxeram mecanismos para garantir sua sobrevivência e também abordar e escrever a história negra dentro dos Estados Unidos e fora dele.

Dorothy Porter Wesley se tornou uma proeminente e conceituada bibliotecária negra, bibliófila e escritora do século XX (FINDLAY, 2001). Dedicou-se por 65 anos $^{3}$ - desde que foi nomeada em 1930 como bibliotecária na Moorland Foundation da Howard University

seja um fato em alguns contextos dos Estados Unidos. Para tanto, quando utilizamos o termo "negra" ou "afro-americana" estamos nos referindo a pessoas de origem africana na diáspora, esta última resultante de crimes contra humanidade.

${ }^{2}$ Termo utilizado por Dorothy Porter Wesley.

${ }^{3}$ Apesar de ter se aposentado como bibliotecária do Centro em 1973, Dorothy continuou sua busca e elaborou uma coleção pessoal sobre estudos negros que, após seu falecimento, foi doada por sua filha ao African-American Research Library and Cultural Center. 
- a unir sua paixão por estudos negros à catalogação e preservação da informação. Sua história profissional foi marcada pela busca em reunir a história da população negra dos Estados Unidos e da diáspora africana (PYNE, 1994; BATTLE, 2007; BLEDSOE, 2018).

Apesar de viver parte de sua vida durante períodos de exclusão de bibliografias e epistemes negras por conta das leis segregacionistas e do racismo instaurado nas instituições profissionais e de ensino, como na macropolítica americana, Dorothy Porter Wesley lutou pela preservação, organização, recuperação e disseminação das informações sobre seu povo. Como resultado, Porter Wesley mudou a visão das instituições sobre as coleções especiais negras e permitiu dar voz às narrativas historicamente silenciadas e invisibilizadas no campo biblioteconômico-informacional (DAGBOVIE, 2004, BLEDSOE, 2018).

Partindo desse contexto, refletimos sobre a profissão bibliotecária e a sua relação com a invisibilização de raça e a promoção do privilégio racial (HONMA, 2017; SILVA, 2020) dentro da produção e organização do conhecimento que consumimos. Nesse sentido, este artigo desenvolve uma reflexão biobibliográfica, fruto de um método epistemológico histórico, crítico-social, sobre a constituição do campo. Trata-se de uma pesquisa que recondiciona o discurso hegemônico de modalidades de constituição epistêmica em Biblioteconomia e Ciência da Informação.

Partindo de outras possibilidades de compreensão das condições críticas de edificação de uma episteme, está em evidência aqui vivências, resistências, corpos e práxis como concretudes que estão no lugar da elaboração científica, teórica, metodológica e profissional do campo. Abre-se aqui, da refundação da bibliografia e da organização do conhecimento no início do século XX, à recompreensão do campo biblioteconômicoinformacional, um exercício teórico-aplicado de democracia documentária (SALDANHA, 2020) como ferramenta de transformação social.

O objeto de estudo é empiricamente fundamentado a partir da centralidade da atuação da bibliotecária negra Dorothy Porter Wesley com dois principais enfoques: 0 primeiro se refere ao seu trabalho para organizar a Coleção do Moorland-Spingarn Research Center da Universidade de Howard elaborada com diversos recursos informacionais da e sobre a população de origem africana nos Estados Unidos e no mundo; o segundo, se dedica a uma breve exploração das limitações da Classificação Decimal de Dewey e as nuances do racismo percebidas e denunciadas por Dorothy Porter Wesley em suas bibliografias, artigos, textos e cartas, contexto este que a direcionou para elaborar A catalogue of the African Collection in The Moorland Foundation Howard University Library (PORTER, 1958). Ambos os movimentos demonstram a aplicação objetiva de uma práxis na episteme do campo biblioteconômico-informacional: ação para transformação através de teorias e de métodos do próprio campo dentro da intervenção na realidade social.

Trata-se, portanto, de uma pesquisa bibliográfica e documental, que adotou como corpus artigos, livros, bibliografias e catálogos produzidos por Dorothy Porter Wesley durante sua atuação bibliotecária, os documentos disponibilizados pelo MoorlandSpingarn Research Center no site da Coleção, assim como estudos de pesquisadores e 
pesquisadoras estudiosas de suas obras. O corpus mencionado data do período de 1939 a 2020.

\section{DOROTHY BURNETT PORTER WESLEY: BIOBIBLIOGRAFIA E ATUAÇÃO DE UMA BIBLIOTECÁRIA NEGRA}

Filha de pais incentivadores que priorizavam a educação, Dorothy Burnett Porter Wesley foi fortemente influenciada na busca pela posição acadêmica, sendo uma de suas principais metas a conquista do ensino superior e pós-graduação, algo inacessível para as mulheres negras à época (UZELAC, 2010; BOTNICK, 2014). Conforme Pero Gaglo Dagbovie, em seu artigo Black Women Historians from the Late 19th Century to the Dawning of the Civil Rights Movement, historicamente, mulheres afro-americanas enfrentaram uma significativa oposição nos séculos XIX e XX, "na busca e obtenção de educação superior, especialmente em programas de pós-graduação de elite" (DAGBOVIE, 2004, p. 241), o que contribuiu para sistemática exclusão de mulheres negras da cultura acadêmica afro-americana e americana.

Dorothy Burnett Porter nasceu em Warrenton na Virgínia, em maio de 1905, filha de Hayes Joseph Burnett, médico graduado pela Howard University, e Roberta Ball, extenista profissional da New Jersey Tennis Association. Como grande parte das mulheres negras de classe média, viveu num bairro hegemonicamente branco em New Jersey, e desde o ensino fundamental direcionou seus esforços para se tornar professora por influência da mãe, Roberta, haja vista o entendimento de que a educação era o caminho para emancipação da comunidade negra (BATTLE, 2007; SIMS-WOOD, 2014).

Para tanto, frequentou instituições públicas de ensino etnicamente mistas, iniciando na Miner Normal School (posteriormente chamada de Miner Teachers College) em Washington, uma escola conhecida por se dedicar ao ensino de meninas negras no século XIX. Posteriormente, Porter Wesley cursou a escola secundária Montclair High School, e logo depois foi trabalhar na Howard University, na qual prestava serviços como assistente de biblioteca na antiga Biblioteca Carnegie [precursora da Biblioteca dos Fundadores, onde o Centro de Pesquisa Moorland-Spingarn agora, em 2021, está instalado] enquanto cursava Mestrado em Biblioteconomia na Columbia University (SCARUPA, 1990). Além disso, Dorothy concluiu a pós-graduação e se tornou a primeira mulher negra a obter o título de Mestra em Biblioteconomia pela Columbia University em 1932, período em que iniciou a colecionar materiais acerca da história da população africana e sua diáspora (BATTLE, 2007; UZELAC, 2010; BOTNICK, 2014; SIMS-WOOD, 2014).

As experiências de Dorothy se iniciaram em 1928 na Biblioteca da Howard University quando foi nomeada bibliotecária-chefe. Na década de 1930, a Howard University se tornou uma das principais instituições acadêmicas destinadas às elites negras e seus descendentes. "Sessenta e cinco anos longe da escravidão, a primeira geração de graduados de Howard abriu caminho para uma nova safra. Entre esse número estava Dorothy Porter" (BLEDSOE, 2018, s.p., tradução nossa). Faz-se importante demarcar 
que esse é um contexto de forte recessão econômica nos Estudos Unidos, o que causou fome e miséria no país, afetando sobremaneira a sociedade afro-americana. Dois anos depois de se tornar bibliotecária da Universidade, em 1930, iniciou a curadoria, reorganização de informações, administração e catalogação da Coleção do educador e ex-ministro, Jesse E. Moorland, doada com mais de três mil títulos à Howard University (BOTNICK, 2014). Essa Coleção foi o início do que hoje conhecemos como Moorland-Spingarn Research Center, localizado na Biblioteca dos Fundadores da Howard University (HOLMES, 1918). Conforme enfatiza Thomas Battle (2007, s.p., tradução nossa):

\begin{abstract}
Enquanto o Dr. Jesse E. Moorland recebe o merecido reconhecimento de ter fornecido à Howard University uma base para a pesquisa histórica sobre os negros, é Dorothy Porter Wesley quem merece o crédito por transformar o potencial do presente de Moorland e outros materiais relacionados em um repositório amplamente aclamado como um dos melhores na época de sua aposentadoria em 1973. Acadêmicos e bibliotecários continuam a se maravilhar com o sucesso que ela conseguiu alcançar em circunstâncias, muitas vezes, difíceis. Tudo o que o MoorlandSpingarn Research Center é hoje é o resultado do trabalho de base estabelecido por ela.
\end{abstract}

Em 1933, Porter Wesley publicou um panfleto informativo que evidenciava quais eram os objetivos da Fundação Moorland, sendo eles:

[...] acumular, registrar e preservar material pelo e sobre o Negro; ajudar estudantes interessados na vida negra a buscarem a exploração acadêmica do material da coleção; para incutir orgulho racial e consciência racial na juventude negra; e fornecer uma grande biblioteca de referência em todas as fases da vida do negro (BOTNICK, 2014, p. 23, tradução nossa).

O Moorland-Spingarn Research Center é "uma unidade administrativa que contém as bibliotecas, arquivos da universidade, museu e coleções especiais adicionais na Howard University" (BLEDSOE, 2018, s.p., tradução nossa). Atualmente, este Centro possui uma das "coleções mais abrangentes da história e memorabilia afro-americana, negra e africana encontrada em qualquer lugar do mundo" (LIBRARY OF CONGRESS, 2020, s.p., tradução nossa). Em seu interior, há diversos recursos informacionais nos mais variados suportes, dentre os quais podemos citar livros, panfletos, periódicos, fotografias, documentos pessoais, manuscritos, partituras de música, artefatos e outros materiais que documentam a história, cultura e experiências dos africanos e seus descendentes na África, América Latina, Caribe, Estados Unidos, e outros lugares do globo (BATTLE, 2007; LIBRARY OF CONGRESS, 2020; TRAPP, 2020). 
Em seus relatos, Porter Wesley evidenciou a crescente demanda de estudantes, professoras, bibliotecárias interessadas nos estudos negros. Tal interesse se deve, em grande parte, ao "internacionalismo negro" evidenciado à época, uma onda surgida no período entre guerras "mundiais" que envolvia disputas por narrativas entre garveyistas, socialistas negros e integracionistas da classe média na década de 1920 e abrangeu diversas posições ideológicas afrocentradas (HOLTON, 2007). Nota-se que, à mesma época, o contexto dos anos 1930, pode-se observar uma intensa movimentação no campo entre Oriente e Ocidente, da obra de Ranganathan na Índia ao desenvolvimento no território americano do doutorado em Biblioteconomia no contexto da Escola de Chicago. A práxis de Dorothy integra, de modo crítico e transformador, essa historicidade do campo, com similar relevância teórica e histórica.

Dorothy encontrou em David Ruggles, um abolicionista negro e, "provavelmente, o primeiro negro colecionador de livros", uma visão dos direitos humanos com lições importantes para reconstruir o legado negro no pós-Segunda Guerra. Em seu artigo intitulado "David Ruggles, an apostle of human rights", Dorothy infere que Ruggles assim como John Brown, Harriet Tubman, Stephen Myers, Lewis Hayden, e tantos outros participantes da luta abolicionista - não recebeu o reconhecimento que deveria pela sua contribuição para o povo negro americano (PORTER, 1943, 1969; KELLEY, 1999).

Uma de suas ações em prol do reconhecimento da bibliografia sobre estudos negros e africanos foi o curso realizado a convite de Wallace Van Jackson, bibliotecário da Virginia Union University. Dorothy foi convidada a participar da $58^{\text {a }}$ Conferência Anual da American Library Association (A.L.A.) em 1936, na qual ministrou o workshop sobre os "Bibliographic Resources for a Study of the American Negro". O referido curso trouxe o tema da bibliografia negra, a listagem de obras compiladas por Porter Wesley até aquele momento, assim como a sua prática para classificar os materiais da Coleção (BOTNICK, 2014).

Com vasta experiência profissional, a bibliotecária prestou consultoria para a Fundação Ford e para a Biblioteca Nacional em Lagos, Nigéria, entre os anos de 1962 e 1964, assim como atuou ativamente no $1^{\circ}$ Congresso Internacional de Africanistas em Acra, Gana. Em seu ensaio "The Negro in the Brazilian Abolition Movement", publicado em 1952, Dorothy demonstra seu interesse na população negra brasileira, o que mais tarde resultaria em outro estudo publicado em 1978 intitulado "Afro-Braziliana: A Working Bibliography” (PORTER, 1978; KELLEY, 1999; UZELAC, 2010; TRAPP, 2020).

Como parte das evidências de uma análise biobibliográfica, a trajetória aqui investigada demonstra a lista de distinções e reconhecimentos. Ao longo da sua carreira, Porter Wesley foi homenageada com diversas premiações. Em 1944, ganhou a bolsa Julius Rosenwald para pesquisa na literatura latino-americana. Em 1973, recebeu a bolsa de estudos e viagens da Fundação Ford que a levou para a Escócia, Irlanda, Inglaterra e Itália. Em 1974, ganhou o prêmio Distinguished Alumni da Howard University e, em 1980, o Prêmio Conover-Porter, o prêmio de maior prestígio para obras publicadas de bibliografia ou referência sobre a África. Em 1989, Porter passou a ser bolsista no W. E. 
B. Du Bois Institute da Harvard University e, naquele mesmo ano, o Moorland-Spingarn Research Center deu início a série anual de palestras Dorothy Porter Wesley. Em 1994, o presidente Bill Clinton a presenteou com o Prêmio Charles Frankel do National Endowment for the Humanities (UZELAC, 2010).

Assim como outras bibliotecárias negras, Dorothy Porter Wesley foi considerada um recurso humano essencial para época, visto que durante sua carreira atuou publicando e coletando estudos sobre a história e pensamento afro-americanos. Juntamente com a estudiosa Shirley Graham Du Bois, fez uso de suas habilidades acadêmicas para produzir estudos bibliográficos históricos influentes e, assim, elevar a história negra a níveis nunca antes alcançados (DAGBOVIE, 2004). Em seu rol de principais livros e bibliografias elaboradas, Dorothy Porter Wesley publicou: "Documentation on the AfroAmerican: Familiar Sources" (1939), "North American Negro Poets: a bibliographical checklist of their writings, 1760-1944" (1945), "Bibliography and Research in AfricanAmerican Scholarship" (1976), "Afro-Brasilliana: a working bibliography” (1978), "Early negro Writing, 1760-1837” (1995), entre muitos outros. A construção de catálogos e instrumentos de classificação também fez parte de sua atuação bibliotecária, sobretudo pela lacuna deixada por Dewey na construção da Catalogação Decimal que leva seu nome. A seguir, exploraremos essa faceta.

\section{AS FACETAS DO RACISMO NOS INSTRUMENTOS DE CLASSIFICAÇÃO: (IN)VISIBILIDADE NEGRA NA CLASSIFICAÇÃO DECIMAL DE DEWEY, EXPANSIVE CLASSIFICATION E CLASSIFICAÇÃO DA LIBRARY OF CONGRESS}

$\mathrm{Na}$ historiografia dos estudos informacionais, há pesquisas e reflexões críticas que apontam, dentre outros, a eugenia presente em esquemas de classificação (TENNIS, 2012), a rejeição da história negra e a presença da violência epistêmica realizada contra os conhecimentos de povos negros em sistemas de classificação (ADLER, 2016, 2017), a discussão sobre a hierarquização de sujeitos racializados nas classificações de Cutter e de Dewey (OLSON, 1998; 2001; 2004), a proposta de “desracialização" da CDD via Teoria Crítica Racial (FURNER; DUNBAR, 2004; FURNER, 2007), a reprodução da estigmatização de pessoas com deficiência em sistemas de organização do conhecimento (ADLER; HUBER; NIX, 2017), e outros enfoques.

Melissa Adler, em seu artigo "Classification Along the Color Line: Excavating Racism in the Stacks", infere que a "violência sistêmica é fundamentalmente um problema da classificação" (ADLER, 2017, p. 3, tradução nossa). Em publicação anterior, a autora havia advogado em prol da reparação taxonômica (ADLER, 2016), haja vista que os marcos históricos da sociedade estadunidense do século XIX estiveram baseados na classificação para fundamentar seus projetos, organizar livros e o conhecimento em categorias étnicas, raciais e religiosas, visando o controle social de todo o país (ADLER, 2016, 2017). 
Para demonstrar como a classificação está embasada em viés racializado, haja vista que "todo esquema de classificação é uma representação objetiva de um ponto de vista subjetivo de seus construtores, que compartilham as perspectivas e ideologias das populações com as quais se identificam" (FUNER, 2007, p. 154, tradução nossa), Adler (2017) analisou três classificações: a Expansive Classification ${ }^{4}$, de Charles Cutter, a Dewey Decimal Classification, de Melvil Dewey e a Classificação da Library of Congress.

Referente à Expansive Classification, Hope Olson (2001, p. 643) já havia criticado a lógica da Classificação de Cutter, pois esta buscava atender "o interesse singular do público". Entretanto, o

[...] público singular de Cutter não é 'todos os membros da comunidade'. É uma parte específica da humanidade que compartilha interesses culturais, sociais ou políticos. Essa comunidade idealizada exclui indivíduos e grupos que não compartilham seus interesses (OLSON, 2001, p. 643).

Dessa forma, Cutter assumiria que "o público dita o vocabulário de uma linguagem universal para representação de informações" (OLSON, 2001). Ou seja, a intenção estava em universalizar - via presunção de uma universalidade que inexiste - ao mesmo tempo em que impunha perspectivas únicas de olhar e classificar o mundo e o Outro.

Na análise da sétima versão da Expansive Classification, de Charles Cutter, Melissa Adler apontou que esta serviu de modelo para outros sistemas de classificação, inclusive para a Classificação da Biblioteca do Congresso Americano, informação corroborada pelas pesquisadoras Alice Príncipe Barbosa e Maria Requião Piedade (BARBOSA 1969; PIEDADE, 1983; ADLER, 2017). O sistema criado por Cutter seguia abordagens de teorias evolucionistas e evidenciou o pensamento do autor em relação à raça:

Por exemplo, a edição de 1902 da sétima expansão classifica os "negros" em três locais: etnologia (PY) na seção de Antropologia; e educação de classes especiais e trabalho escravo, ambos nas Ciências Sociais. Outra classe - F8339, definida como "Controvérsia da escravidão", foi arquivada na história americana, mas não mencionou uma categoria racial. [...]

A seção PY estava especificamente preocupada com Etnologia - o ramo da Antropologia que lida com raça e etnia. Foi organizado em uma variedade de subdivisões, impulsionado em parte pelo esquema teórico no qual as classificações de raça e etnia foram baseadas. Por exemplo, a subclasse PYE fornece uma metataxonomia para "Etnografia (raças de homens)", indicando que a raça pode ser determinada de todas essas maneiras diferentes: "Agrupamento somatológico (físico)", "Agrupamento geográfico", "Agrupamento linguístico", "Por instituições e organização social”,

4 Versão da Classificação de Cutter analisada pela autora disponível em: https://babel.hathitrust.org/cgi/pt?id=uc1.C027192510\&view=image\&seq=5 Acesso em: 20 jan. 2021. 


\begin{abstract}
"Por artes e cultura", "Por sistemas musicais" e "Por mitologia e religião." [...]

A seção de Ciências Sociais da sétima expansão abrangia economia, sociologia, educação e direito. [...] "Negros, libertos" foi classificado no IZ dentro da educação, junto com uma série de outras populações marginalizadas. Observe que isso está no final da classificação, diferente de IK-IY - aulas dedicadas a tópicos relacionados à educação (pedagogia, matérias escolares, níveis de escolaridade, etc.) para um suposto homem branco, "apto", população americana proprietária. Da mesma forma, trabalhos sobre "Escravidão nos EUA" foram classificados na seção HI como uma categoria dentro de trabalho e produção (ADLER, 2017, p. 6-7, tradução nossa).
\end{abstract}

A partir das análises realizadas nas classes e subdivisões, segundo exemplificado nos trechos acima, a autora concluiu que no sistema de Cutter "os afro-americanos eram objetos de estudo e interesse na medida em que informavam o comércio, as teorias de raça e evolução social e uma conceituação estritamente definida da moralidade pública" (ADLER, 2017, p. 10, tradução nossa).

Enquanto isso, na Classificação de Dewey5 ${ }^{5}$, Melissa Adler (2017) apresenta como a referida Classificação reflete a visão do autor no que se refere à população afroamericana. Conforme sua análise, as edições inicialmente lançadas da Classificação Decimal de Dewey seguiam o sistema de Cutter, especialmente no que concerne à raça, antropologia e escravidão. Importante frisar que embora a classificação de Dewey seja anterior à classificação de Cutter, a análise realizada por Adler (2017) tem validade na medida em que recaiu também sobre as edições posteriores (atualizadas) ${ }^{6} \mathrm{da}$ classificação de Dewey.

Na classificação, "a opressão ocorre por meio de um preconceito aberto ou insidioso que pode potencialmente negar o acesso por meio de estruturas criadas por e para uma classe dominante (FOX, 2015, p. 17, tradução nossa). No índice de assuntos da edição impressa publicada em 1876 e avaliada por Adler (2017), a racialização de sujeitos negros estava presente na Classificação: “os trabalhos sobre 'Negros' devem ser classificados em dois lugares: 573 , designado para "História Natural do Homem" dentro da Biologia, ou em 326, reservado para "Escravidão" dentro da seção de ciência política” (ADLER, 2017, p. 15, tradução nossa). Em 1919 houve atualização7 da CDD, e ainda assim, a visão do autor sobre pessoas africanas e afro-americanas permanecia:

[...] a seção 573 é dividida em categorias como 'Cor do homem', que é seguida por 'Antropometria', 'Craniologia', ‘Anões e gigantes' e

\footnotetext{
5 Versão analisada pela autora disponível em: https://archive.org/details/deweydecimal10dewe Acesso em: 20 jan. 2021.

6 Melissa Adler realizou a análise em 2015 no site WebDewey, disponível no link: https://dewey.org/webdewey/login/login.html Acesso em: 20 jan. 2021.

7 Versão da Classificação de 1919 está disponível aqui: https://archive.org/details/deweydecimal10dewe/page/8/mode/2up Acesso em: 20 jan. 2021.
} 
'Monstruosidades'. Em 572, vemos associações semelhantes, visto que se relacionam com entendimentos antropológicos/etnológicos de raça. (ADLER, 2017, p. 15, tradução nossa).

Embora revisitada sistematicamente, Adler advoga que a CDD promove em seus tópicos e subdivisões a "primazia das raças europeias", a qual é sustentada na organização de grupos étnicos e nacionais específicos. A análise de Adler (2017) foi de que subdividir negros dos Estados Unidos como "Outros grupos étnicos e nacionais" os distancia da categoria de povo estadunidense, ao mesmo tempo em que fornece a falsa percepção de que para ser americano deve ser descendentes de europeus (OLSON, 2001; 2004).

Para Dewey, a padronização sempre foi mais urgente do que a mudança. Assim, os sistemas alternativos à CDD feitos por bibliotecários para adequar a seus acervos eram mantidos fora de circulação por Dewey e seus seguidores. Tais proibições eram embasadas no pensamento do autor de que "ferramentas de categorização eram úteis ao invés de epistemológicas" ou, como inferiu na edição de 1951, "a classificação do livro é essencialmente funcional, um meio de localização, não um sistema filosófico" (DEWEY, 1951 apud HELTON, 2019, p. 104).

Com base nas afirmações acima, Dewey parecia querer se furtar da discussão filosófica, propagandeando sua própria classificação como uma função utilitária. No entanto, estudos como os de Leidecker (1946), Graziano (1959), La Montagne (1961), Wiegand (1996, 1998), Olson (2011), Sales (2017) e Ferreira e Sales (2018) mostram que Dewey, ao elaborar seu sistema de classificação, valeu-se de classificações já existentes e em uso no século XIX, que, por sua vez, eram expressamente pautadas nas classificações lógico-filosóficas de Bacon e Hegel, especialmente a classificação de livros de William Torrey Harris de 1870. Se o encadeamento lógico dos assuntos não era o mote da discussão para Dewey, que dava primazia à utilidade e à praticidade de sua classificação, isso se devia ao fato de ele ter se apropriado de ordenações classificatórias de outros autores, como Harris e os professores do Amherst College (Massachusetts), onde Dewey estudou, cujos professores sofriam forte influência hegeliana.

Por fim, quanto à Classificação da Biblioteca do Congresso Americano ${ }^{8}$, esta foi influenciada pelos sistemas anteriormente citados. Adotou, inclusive, semelhante estrutura evolutiva de Cutter, mas realizou algumas alocações diferentes de classes, especialmente na exclusão da educação da seção de Ciências Sociais à época de seu rascunho de 1901 (MILLS, 1960; BARBOSA, 1969; LENTINO, 1971; FOSKETT, 1973; PIEDADE, 1983; ADLER, 2017). Nesta oportunidade, foi elaborada uma divisão para "Classe de pessoa" em Sociologia, nas quais as divisões eram: IZA. Cego e surdo e mudo; IZB. Cego; IZC. Surdo e mudo; IZE. Débil Mental; IZI. Índios; IZK. Criminosos; IZN. Negros, libertos; IZP pobre, O. (LC, 1901 apud ADLER, 2017, tradução nossa). A autora

8 Versão da Classificação da Biblioteca do Congresso Americano analisada pela autora disponível em: https://babel.hathitrust.org/cgi/pt?id=mdp.39015030534823\&view=image\&seq=3 Acesso em: 20 jan. 2021. 
infere que a Classificação da LC segue o sistema de Cutter no que se refere aos afroamericanos, pois os coloca em trabalho ou classe especial (ADLER, 2017).

A seção H foi impressa em 1910 e combina seções da Classificação de Cutter, inclusive classificando afro-americanos como "libertos" e/ou "negros", os quais podiam ser classificados em três locais: Sociedades secretas (Maçonaria entre negros), Sociedades raciais (negro) e Patologia social, Bem-estar social público, Criminologia (Classes especiais) - por raça - negros (LC, 1910 apud ADLER, 2017). Em 2015, Adler visitou o site online da Classificação da LC, e de acordo com a autora: "HS875-891 é definido como 'Maçonaria entre negros'; HS2226-2230 (movido ligeiramente do local HS2251-2265) é 'Negros' em 'Sociedades raciais'; e HV3181-3185 é 'Africano americano' como uma 'raça ou grupo étnico especial’ em ‘Proteção, assistência e auxílio'. Não mudou muito” nesse período de mais de 100 anos (ADLER, 2017, p. 21, tradução nossa).

Dessa forma, após as três análises das três Classificações, Adler afirma que, no que se refere à raça, os referidos sistemas continuam a sustentar e complementar as conceituações uns dos outros, assim como os discursos normativos dominantes. Dessa forma, a invisibilidade da raça nesses instrumentos demarca que as hierarquias antes criadas continuam invisíveis e radicadas nos sistemas de recuperação de informação, bibliotecas e comunidades (ADLER, 2017).

Objetivamente, a evidência das classificações demonstra o apagamento, a exclusão e/ou a opressão no plano racial, sendo cada classe mantenedora e simultaneamente multiplicadora de um ou dos três aspectos destacados. Compreendendo o papel das classificações dentro do complexo sistema metalinguístico de uma sociedade, no contexto das sociedades afro-americanas tem-se aqui a demarcação do exercício racista de construção social do Século XX em uma de suas empirias. Do local para o global, os impactos para concepção de uma utopia democrática são enormes quando avaliados os resultados das observações dos parágrafos anteriores: descontextualizada dentro da própria sociedade para a qual é concebida, cada classificação aqui iluminada tem sua repercussão internacional, centralmente o modelo deweyano, ampliando em um plano mundial o mal representado pelas decisões do sistema no solo local.

\section{INSURGÊNCIA DAS BIBLIOGRAFIAS NEGRAS: COLETA E ORGANIZAÇÃO DA HISTÓRIA AFRICANA E NEGRA}

Na seção que se segue, retomaremos a atuação de Dorothy Porter Wesley, em especial, sua denúncia do preconceito racial presente na CDD e a insuficiência do referido sistema para abarcar o conhecimento negro. Por fim, apresentamos como a bibliotecária conseguiu vencer esse obstáculo a partir da elaboração do $A$ Catalogue of Books in the Moorland Foundation (1939) e do A catalogue of the African Collection in the Moorland Foundationn Howard University Library (1958) para catalogação da Coleção. 
A construção demonstra, como será observado, o modo como a metodologia bibliográfica estabelece o potencial de práxis, ou seja, de ação social com intencionalidade objetiva orientada para transformação. Como teoria e método, conceito e ferramenta, a bibliografia negra e suas múltiplas experiências aplicadas demonstra-se como forma de luta e de resistência, de visibilidade e de humanidade.

\section{A insuficiência da Classificação Decimal de Dewey para abarcar a história negra}

Um dos papéis das bibliotecas, arquivos e centros que abrigam coleções negras está em atuar como um reservatório da intelectualidade negra, servindo como uma historiografia física do pensamento negro. Esse papel se amplia à medida que são adotadas abordagens multifacetadas e parcerias institucionais que permitem a ampla pesquisa dessas informações (BLEDSOE, 2018).

Para Kara Bledsoe (2018, s.p., tradução nossa), coleções negras (Black collections) "contêm uma riqueza de conhecimento que apoia, aumenta e inspira não apenas os estudos negros, mas potencialmente também qualquer investigação pertencente a pessoas da diáspora africana". A importância de se construir coleções negras foi evidenciada no trabalho realizado por Dorothy Porter Wesley, especialmente ao compreender que tais coleções são fontes para pesquisas futuras sobre identidade negra no campo biblioteconômico-informacional e diversas áreas do conhecimento.

A Howard University começou sua coleção de títulos sobre África nos primeiros anos de sua biblioteca criada pelos fundadores da Universidade. Interessados em missões estrangeiras, os mesmos doaram biografias de missionários e livros de viagem para fazerem parte do acervo. Essas doações de livros sobre África continuaram a chegar até que em 1914, Jesse Edward Moorland doou sua coleção particular para a biblioteca. Dentro de sua coleção vieram mais do que livros, foram doadas peças de arte, manuscritos escritos por africanos direcionados ao Jesse Moorland, entre outros (PORTER, 1959). Foi a partir do recebimento dessa coleção que o Conselho de Curadores da Universidade decidiu criar a Fundação Moorland, "uma biblioteca da vida e história negras" (PORTER, 1959).

A Fundação tinha o objetivo de coletar e preservar a produção impressa e não impressa escrita por pessoas africanas, negras e da diáspora. A partir da oferta de cursos sobre cultura e civilização de povos negros em África, a biblioteca começou a organizar uma coleção especial sobre a população negra visando atender às necessidades curriculares sobre África. Simultaneamente, a coleção continuou a expandir seu acervo por intermédio de compra, doações e transferência de livros da Biblioteca Central da Instituição (PORTER, 1959). Com isso, planejou e implantou uma Sala de Leitura, especialmente pensada para os interessados em história, literatura e arte negra, a qual permitiu ainda que as obras se mantivessem na Coleção e sempre disponíveis para aquelas pessoas que buscassem acesso aos materiais, haja vista que não estavam disponíveis para empréstimo, somente para consulta local (LUBIN, 1973; HELTON, 2019). 
As contribuições de Dorothy Porter Wesley - para além da coleta, organização do acervo e compilação de bibliografias negras - estiveram vinculadas à consciência crítica sobre o preconceito racial na sociedade americana, em especial, no entendimento de que as bibliotecas reproduzem o racismo institucional (TRAPP, 2020).

Durante seu trabalho como curadora da Coleção Moorland-Spingard, denunciou o preconceito racial presente na Classificação Decimal de Dewey (SIMS-WOOD, 2014; NUNES, 2018). Embora não se possa negar a revolução do sistema criado por Dewey, também não é possível esquecer que o referido sistema refletiu os preconceitos de seu fundador e de sua época, conforme infere Botnick (2014). Utilizada pelas(os) bibliotecárias(os) para classificar os livros, à época, a CDD "não tinha uma maneira válida de acomodar os livros que tratavam da experiência negra" (SCARUPA, 1990, p. 3, tradução nossa)

Em entrevista à Harriet Jackson Scarupa (1990), Dorothy Porter Wesley apontou sua resistência em utilizar a CDD devido às invisibilidades encontradas: "No sistema, tudo relacionado ao Negro era classificado em '325', que era o número para 'colonização', ou '326', que era o número para 'escravidão'” (PORTER, 1990, p. 3, tradução nossa). Sua indignação estava no fato de que ambas classes não representavam a miríade do conhecimento e da história negros. Em "muitas das bibliotecas brancas, que visitei mais tarde - cada livro, fosse um livro de poemas de James Weldon Johnson, que todos sabiam que era um poeta negro, tinha que estar na 325. E isso era estúpido para mim" (PORTER, 1995, p. 12, tradução nossa).

Ainda, enfrentou resistência dentro da própria Howard à sua decisão de criar um outro modo de catalogar o conhecimento negro, haja vista as insuficiências supracitadas: "A mulher responsável pela classificação decimal de Dewey em Howard não conseguia ver por que eu queria desenvolver outra coisa, por que não queria colocar um livro de poesia de James Weldon Johnson em '325' ou '326' - que era ridículo!". (PORTER, 1990, p. 3, tradução nossa).

Dorothy havia contatado e solicitado à Jennie Dorcas Fellows ${ }^{9}$, sucessora de Dewey, alterações no sistema em decorrência dos problemas encontrados, pois o mesmo era limitante para classificar tudo que fosse produzido por ou sobre africanos, afroamericanos e da diáspora africana (BOTNICK, 2014; OMBUD, 2020). A sugestão de Dorothy era que o sistema fosse expandido visando acomodar os materiais da Coleção. Fellows respondeu sugerindo "a possibilidade de usar, em sua maior parte, números regulares [Dewey] para o tópico em questão, com alguma designação de prefixo, por ex. N para Negro, para indicar o ângulo especial a partir do qual o assunto estava sendo considerado" (FELLOWS apud BOTNIK, 2014, p. 35, tradução nossa).

Em $\operatorname{cartas}^{10}$ trocadas entre elas, Fellows recusou o pedido de Dorothy Porter Wesley para mimeografar o Tentative Supplementary Classification Scheme, informando que "isso estaria totalmente fora de questão, a menos que o tivéssemos aprovado em

${ }^{9}$ A bibliotecária modificou o sobrenome para Dorkas, como é atualmente conhecida.

${ }^{10}$ Dorkas Fellows to Dorothy Porter, December 6, 1934, box 5, Wesley Papers. 
detalhes" (FELLOWS apud HELTON, 2019, p. 106, tradução nossa), haja vista que essa alteração "resultaria na destruição de toda padronização" criada por Dewey (FELLOWS apud HELTON, 2019, p. 106, tradução nossa). Após a recusa, Dorothy recebeu uma advertência da American Library Association avisando que se compartilhasse seu esquema de classificação adaptado com outras(os) bibliotecárias(os) seria acusada de violar os direitos autorais da obra de Dewey (HELTON, 2019).

Outro episódio de resistência esteve na divergência sobre o que deveria ser incluso no acervo da Coleção. Na percepção de Dorothy Porter Wesley, a Coleção não poderia se limitar somente a livros e periódicos, mas também deveria incluir cartas, diários, fotografias, partituras, discos, recortes de jornais, artefatos e similares (SCARUPA, 1990). No entanto, haviam pessoas da Howard que discordavam de sua perspectiva:

Havia um tesoureiro que achava que eu não deveria comprar música [...] Então, tive que fazer isso secretamente. Eu compraria algo como 'Imaginary Ballet', de Samuel Coleridge Taylor - que era música - e listaria junto com os livros. Ele não teria tempo de ler essas coisas de qualquer maneira. (PORTER, 1990, p. 3, tradução nossa)

Dessa forma, Dorothy Porter Wesley não só conseguiu adquirir os recursos informacionais que desejava e também desviar do racismo institucional na organização, como em 1958 a Coleção compreendia 65.386 itens catalogados, dos quais eram livros, panfletos, periódicos, jornais, manuscritos e microfilmes e centenas de livros não catalogados ou não indexados, materiais periódicos e manuscritos, além de fotografias, músicas e outros itens não impressos (PORTER, 1969).

Os dados biobibliográficos e a luta através da própria bibliografia demonstram a fundamentação política e crítica de uma linha de constituição da própria epistemologia do campo informacional. Um dos nascimentos do campo, em seu próprio sentido metametodológico, encontra na práxis bibliográfica negra feminina sua forma de verdade: uma cientificidade pautada na transformação social e na denúncia dos crimes contra humanidade tecidos pela própria metametodologia que é reconstruída no decurso da luta.

\section{A Catalogue of the African Collection in the Moorland Foundation Howard University Library: Organizando o Conhecimento Negro}

Para localizar materiais sobre literatura e história negra acumulados na Howard University desde sua fundação em 1867, a colecionadora de bibliografias Dorothy Porter Wesley rastreou e vasculhou as pilhas de materiais em todos os cantos das bibliotecas central e setoriais da Instituição e os levou para a Coleção (ZALUDA, 1998; HELTON, 2019). Constatou que nas listas de autoridades usadas por catalogadores, existiam poucos nomes de autores negros, e que nos cabeçalhos de assuntos e 
também nas taxonomias "universais" era frequente a omissão de tópicos negros (KELLEY, 1999; HELTON, 2019).

Decidiu, então, utilizar suas habilidades bibliotecárias para iniciar um novo catálogo de fichas e estabelecer uma classificação própria. Buscou entender como unir os textos e artefatos coletados via lógica da negritude, assim como empreendeu uma taxonomia radical que buscava fornecer textos negros a leitores negros que tinham interesse em suas histórias e ancestralidade (PORTER, 1958; HELTON, 2019).

Dorothy decidiu ignorar a indicação de Fellows sobre a CDD e fez sua própria classificação inspirada no catálogo de classificação da biblioteca do Oberlin College e na Classificação de Dewey:

\footnotetext{
Eu simplesmente comecei a basear tudo sobre a literatura e história negra onde quer que ela caísse na classificação decimal regular de Dewey - se fosse um livro sobre os negros na guerra revolucionária, teria o mesmo número de 'Guerra revolucionária', por exemplo. Em seguida, apenas colocamos um ' $\mathrm{M}$ ' na frente dele, para Moorland. Era muito simples, sabe, muito simples. (PORTER, 1990, p. 4)
}

Em 1939, publicou o "Catalogue of Books in the Moorland Foundation" (Figura 1) com o auxílio da Works Progress Administration (WPA). O referido catálogo apresentava a incorporação dos números da classificação de Dewey personalizados para atender aos interesses da bibliotecária de classificar obras de autoria negra e sobre a população africana na diáspora. Ademais, auxiliou bibliotecas que possuíam recursos informacionais sobre a história negra a classificarem os títulos conforme o catálogo realizado por Dorothy Porter Wesley e que até hoje está presente na Coleção (PORTER; HUNTON; WILLIAMS, 1939; HELTON, 2019).

Porter Wesley entendeu o catálogo como um campo de batalha epistemológico que permitiria re-mapear estruturas antes apagadas do conhecimento negro. Por intermédio da reclassificação, foi possível permitir o acesso à toda extensão de informações sobre a escrita negra, africana e da diáspora, livre da noção universal das bibliotecas brancas (HELTON, 2019).

Figura 1. a) A Catalogue of Books in the Moorland Foundation, primeira edição publicada por Dorothy Porter Wesley e equipe publicada em 1939. b) A catalogue of the African Collection in the Moorland Foundation Howard University Library, editado por Dorothy Porter Wesley e equipe de estudantes publicada em 1958. 

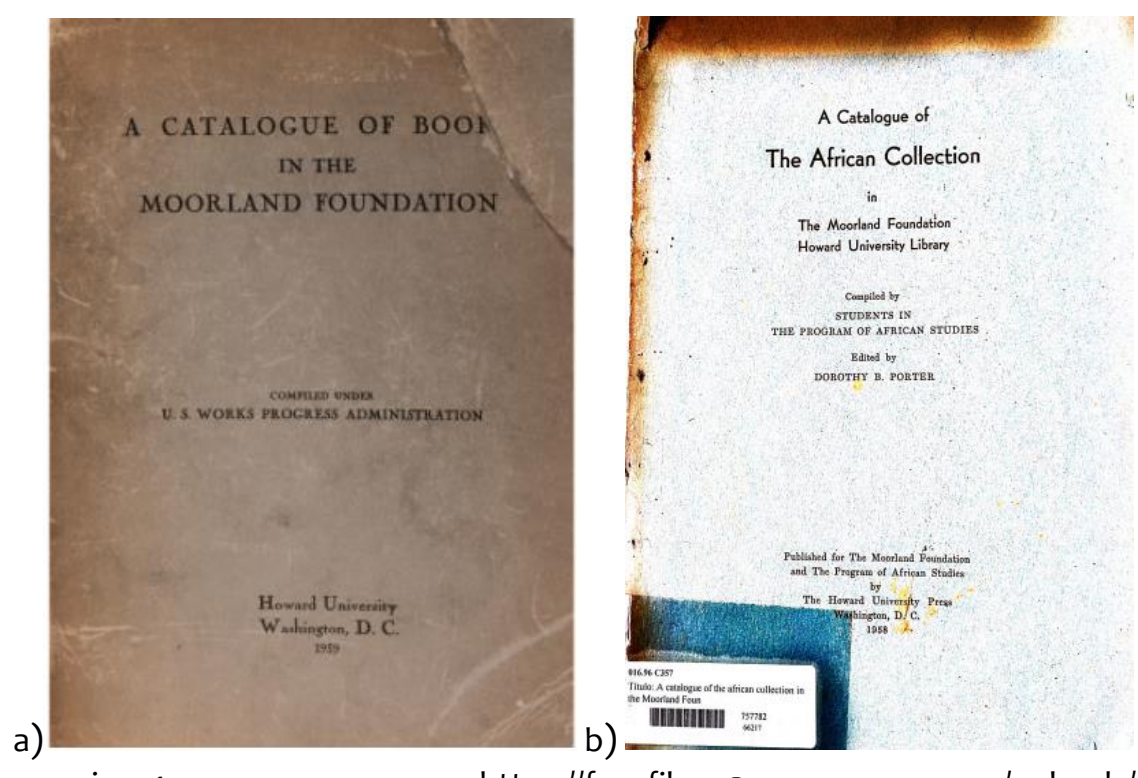

Fonte

imagem a:

https://fws-files.s3.amazonaws.com/uploads/website/ auctions/items/medium/3998331_2.jpg Acesso em: 10 jan. 2020. Fonte imagem b: A catalogue of the African Collection in the Moorland Foundation Howard University Library (1958).

Dorothy elaborou e publicou em 1958 um novo catálogo intitulado "A catalogue of the African Collection in the Moorland Foundation Howard University Library", com apoio financeiro da Fundação Ford. O referido catálogo visou fornecer uma lista dos materiais relacionados à África que estavam catalogados na Moorland Foundation.

Figura 2. Divisão por regiões (exemplo: África Geral - Bibliografias) e assunto (Trabalhos gerais sobre África) A catalogue of the African Collection in the Moorland Foundation Howard University Library, editado por Dorothy Porter Wesley e equipe de estudantes publicada em 1958.
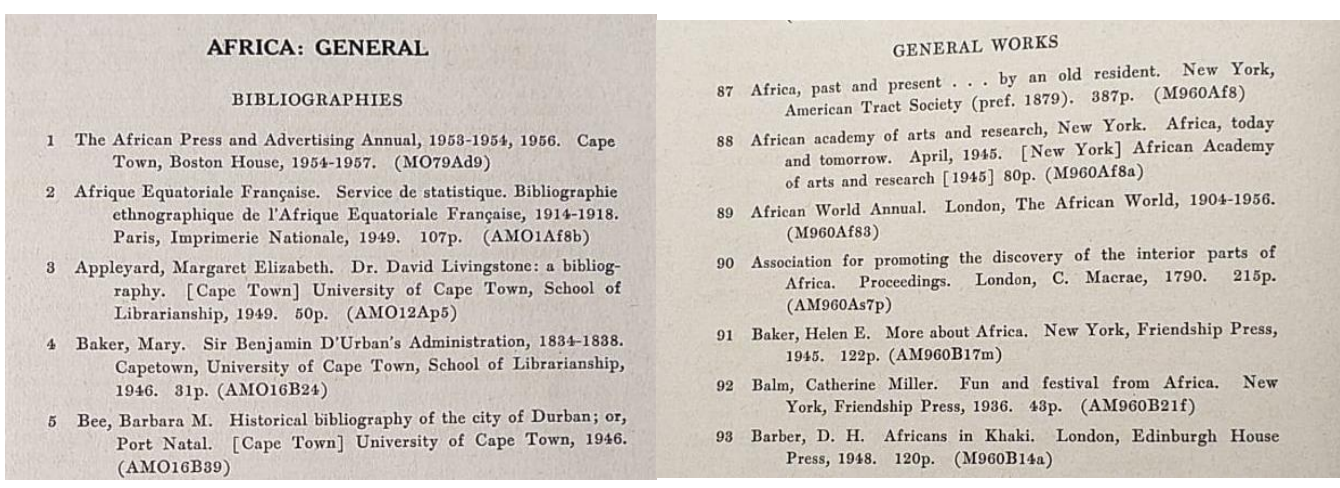

Fonte: A catalogue of the African Collection in the Moorland Foundation Howard University Library (1958).

A edição de 1958 contava com 4.865 entradas de livros incluídas à época via esquema alfabético por países ou unidades políticas, os quais eram por sua vez agrupados em seis regiões principais, como, por exemplo, Norte da África, Nordeste da África, Oeste da África, África Central, Leste da África e Sul da África. O catálogo possui ainda uma seção geral organizada por assunto, a qual precede a listagem de divisões por países (Figura 2) (PORTER, 1958).

Em 1973, ano de sua aposentadoria, a Coleção do Moorland-Spingarn Research Center contava com mais de 195.000 obras inventariadas, catalogadas e classificadas (LUPIN, 
1973). Tudo isso se deve à dedicação e trabalho constantes desta bibliotecária que contribuiu para o amplo conhecimento sobre os Estudos Negros em todo o mundo.

A historicidade aqui relatada nos recoloca no ponto de inflexão da luta por uma democracia documentária na constituição da própria episteme entendida ali, no norte americano, como Library and Information Science. Como estabelecer possibilidades de realização da plenitude democrática prometida pelo discurso patriarcal, machista e branco, dos Estados Unidos da América para o mundo, sem a concepção de uma inflexão dos sistemas de organização do conhecimento como mediações sociotécnicas não partícipes, mas causa e consequência da injustiça social?

Pela via de uma travessia metabibibliográfica, ressignificando continentes e conteúdos, o conhecimento, a pesquisa e a obra de Dorothy Porter Wesley propõe um outro modo, crítico, histórico e social, materialmente tecido, de pensar não apenas a bibliografia e a organização do conhecimento, mas a própria constituição do campo biblioteconômico-informacional. Se ciência e sociedade jamais se dissolvem em unidades polares, distintas e estranhas, a práxis de Dorothy vai, pois, além ainda da constatação epistemológica posta: trata-se de movimento de democracia documentária teórica e aplicada, via intervenção direta, objetiva, na realidade social que segrega, oprime e massacra, na compreensão de como a ciência - aqui, como Biblioteconomia e Ciência da Informação - não podem, fundamentalmente devem, atuar para a transformação social e a humanização de um mundo socialmente corrompido.

\section{CONSIDERAÇÕES FINAIS}

Apresentamos neste artigo a bibliotecária negra Dorothy Porter Wesley e suas contribuições para a Biblioteconomia Negra dos Estados Unidos da América e do mundo. Buscamos dar visibilidade ao esforço de Dorothy ao longo de sua atuação bibliotecária na construção da Coleção Especial sobre a experiência negra da Howard University, a qual é referência para os estudos negros até os dias atuais.

Abordamos como, através de suas habilidades como bibliotecária e perspicácia crítica de uma mulher negra que viveu em período de segregação e invisibilização do conhecimento produzido por e sobre negros, conseguiu influenciar a maneira como os materiais do Centro eram catalogados rejeitando os métodos comumente ensinados e utilizados de maneira costumaz nas bibliotecas americanas daquela época. Contextualizamos como enfrentou as barreiras impostas pela sociedade racista, inclusive dentro da própria Universidade, e construiu um catálogo para organizar informações e materiais disponibilizados na vasta Coleção Especial do MoorlandSpingarn Center. As faces do racismo institucional e as possíveis penalidades que poderia sofrer não foram empecilhos para essa bibliotecária que atuou na organização do conhecimento e encabeçou a criação e o desenvolvimento de uma forma personalizada de catalogar e classificar as contribuições oriundas de autores e autoras negras, e sobre a população africana e diaspórica. 
Dorothy trouxe em sua atuação uma perspectiva crítica, antirracista e decolonial de olhar para o mundo e lutar pela classificação e catalogação justas da literatura, música, artes, bibliografias, poemas e demais produções negras e africanas dentro de diversas áreas do conhecimento. Tal perspectiva nos inspira a direcionar os esforços em sulear pesquisas, bibliotecas e bibliotecárias(os) no aprofundamento dos Estudos Negros em Organização do Conhecimento, ao mesmo tempo em que contribuímos para uma atuação engajada na construção da identidade negra no campo biblioteconômicoinformacional e diversas áreas do conhecimento.

\section{AGRADECIMENTOS}

Agradecemos à Coordenação de Aperfeiçoamento de Pessoal de Nível Superior (CAPES) pela concessão de bolsa de Doutoramento às primeiras autoras - Código de Financiamento 001. Agradecemos ainda, às bibliotecárias da Universidade Federal da Bahia, Valdinéia Barreto e Tatiana Bonfim Sousa, assim como à bibliotecária da Universidade Federal de Minas Gerais, Diná Araujo, pelo auxílio na recuperação do catálogo que foi fonte para esta pesquisa.

\section{FINANCIAMENTO}

A pesquisa foi desenvolvida a partir do fomento do Conselho Nacional para o Desenvolvimento Científico e Tecnológico (CNPq), da Coordenação de Aperfeiçoamento de Pessoal de Nível Superior (Capes) e da Fundação Carlos Chagas Filho de Amparo à Pesquisa do Estado do Rio de Janeiro (Faperj).

\section{REFERÊNCIAS}

ADLER, Melissa. The Case for Taxonomic Reparations. Knowlodge Organization, v. 43, n. 8, p. 630-640, 2016. [Acesso em: 10 out. 2020]. Disponível em: https://www.nomoselibrary.de/10.5771/0943-7444-2016-8-630/the-case-for-taxonomic-reparations-volume43-2016-issue-8

ADLER, Melissa. Classification along the Color Line: Excavating Racism in the Stacks. Journal of Critical Library and Information Studies, n. 1, p. 1-32, 2017. DOI: 10.24242/jclis.v1i1.17

ADLER, Melissa; HUBER, Jeffrey; NIX, Tyler. Stigmatizing Disability: Library Classifications and the Marking and Marginalization of Books about People with Disabilities. Library Quarterly: Information, Community, Policy, v. 87, n. 2, p. 117-135, 2017. DOI: 0024-2519/2017/8702-0004\$10.00

BARBOSA, Alice Príncipe. Teoria e prática dos sistemas de classificação bibliográfica. Rio de Janeiro: Instituto Brasileiro de Bibliografia e Documentação, 1969. 441 p. (Obras Didáticas; 1). 
BATTLE, Thomas C. Moorland-Spingarn Research Center, Howard University. Library Quartely, v. 58, n. 2, p. 143-163, 1988. DOI: 10.1086/601986

BATTLE, Thomas C. Dorothy Porter Wesley: preserver of Black history - AfroAmerican librarian. Diverse Education, 16 jun. 2007. [Acesso em: 10 out. 2020]. Disponível em: https://diverseeducation.com/article/7457/

BELT, Marva E.; HALL, Tomasha P. Dorothy Porter Wesley: A Selected Bibliography. Moorland Spingarn Research Center Publications, 2, 2015. [Acesso em: 10 out. 2020]. Disponível em: https://dh.howard.edu/msrc_pub/2

BLEDSOE, Kara. What Dorothy Porter's Life Meant for Black Studies. Daily, August 22, 2018. [Acesso em: 10 out. 2020]. Disponível em: https://diverseeducation.com/article/7457/

BOTNICK, Julie. I am sure that you know yourself that it is a very good job" The early life and Library of Dorothy Porter. History 215j. The Art of Biography, 2014. [Acesso em: 10 out. 2020]. Disponível em:

http://www.library.yale.edu/ nkuhl/YCALStudentWork/Botnick_Porter_Paper.pdf

BURKETT, Nancy; BURJETT, Randall K. Dorothy Burnett Porter Wesley: Obituaries. American Antiquarian Society, p. 31-35, 1995. [Acesso em: 20 ago. 2020]. Disponível em: https://www.americanantiquarian.org/proceedings/44539475.pdf

DAGBOVIE, Pedro G. Black women historians from the late 19th century to the Dawning of the civil rights movement. The Journal of African American History, v. 89, n. 3, p. 241-261, 2004. [Acesso em: 08 out. 2020]. Disponível em: www.jstor.org/stable/4134077

DEWEY, Melvil. Decimal Classification. Standard 15th ed., Forest Press, 1951.

DU BOIS, William E. B. Reconstruction and its Benefits. The American Historical Review, v. 15, n. 4, p. 781-799, jul. 1910. DOI: 10.2307/1836959

FERREIRA, Verônica de Sá; SALES, Rodrigo de. Confluências filosóficas para os estudos de classificação das artes: aproximações de Bacon, Hegel e Harris. Scire, v. 24, n.1, p. 55-66, 2018.

FINDLAY, James A. Dorothy Porter Wesley (1905-1995), Afro-American librarian and bibliophile: an exhibition. Ft. Lauderdale, Fl.: Bienes Center for the Literary Arts, Broward County Library, 2001.

FORTEN, James; HILTON, John T.; BROWN, William W. Early Manuscript Letters Written by Negroes. The Journal of Negro History, v. 24, n. 2, p. 199-210, 1939. DOI: $10.2307 / 2714449$

FOX, Melodie J. Gender as an 'Interplay of Rules': Detecting Epistemic Interplay of Medical and Legal Discourse with Sex and Gender Classification in Four Editions of the Dewey Decimal Classification. Dissertation (Doctor of Philosophy) - The University of Wisconsin, Milwaukee, 2015. [Acesso em: 10 dez. 2020]. Disponível em: https://dc.uwm.edu/etd/872.

GRAZIANO, Eugene E. Hegel's philosophy as basis for the Dewey Classification Schedule. Libri, v. 9, p. 45- 52, 1959. 
HELTON, Laura. On Decimals, Catalogs, na Racial Imaginaries of Reading. Publications of the Modern Language Association, v. 134, n. 1, p. 99-120, 2019.

HOLMES, Dwight O. W. Fifty Years of Howard University: Part I. The Journal of Negro History, v. 3, n. 2, p. 128-138, 1918. DOI: $10.2307 / 2713486$

HOLTON, Adalaine. Decolonizing History: Arthur Schomburg's Afrodiasporic Archive. The Journal of African American History, v. 92, n. 2, p. 218-238, 2007. [Acesso em: 10 dez. 2020]. Disponível em: https://www.jstor.org/stable/20064181

HONMA, Todd. Foreword. Em: SCHLESSELMAN-TARANGO, G. (ed.). Topographies of Whiteness: mapping whiteness in Library and Information Science. Sacramento: Library Juice Press, 2017. p. 79- 98.

FOSKETT, Antony Charles. A abordagem temática da informação. São Paulo: Polígono; Brasília: UnB, 1973.

FURNER, Jonathan. Dewey Deracialized: A critical race-theoretic perspective. Knowledge Organization, v. 34, n. 3, p. 144-168, 2007. [Acesso em: 10 set. 2021]. Disponível em: https://www.nomos-elibrary.de/10.5771/0943-7444-2007-3144.pdf?download_full_pdf $=1$

FURNER, Jonathan; DUNBAR, Anthony W. The treatment of topics relating to people of mixed race in bibliographic classification schemes: a critical race-theoretic approach. Em: McILWAINE, la C. (ed.). Knowledge Organization and the Global Information Society: Proceedings of the Eighth International ISKO Conference, London, 13-16 July 2004. Würzburg: Ergon Verlag, 2004. p. 115-120.

JORDAN, Casper LeRoy; JOSEY, Elonnie Junius. A Chronology of Events in Black Librarianship. Em: JOSEY; Elonnie Junius; SCHOCKLEY, Ann Allen. (ed.). Handbook of black librarianship. Littleton, Colorado: Libraries Unlimited, 1977.

KELLEY, Robin D. G. ‘But a local phase of a world problem': black history's global vision, 1883-1950. The Journal of American History, v. 86, n. 3, p. 1045-1077, 1999. [Acesso em: 10 jan. 2021]. Disponível em: www.jstor.org/stable/2568605

La MONTAGNE, Leo E. American library classification: with special reference to the Library of Congress. Handen: The Shoe String Press, 1961.

LEIDECKER, Kurt F. Yankee teacher: the life of William Torrey Harris. New York: The Philosophical Library, 1946. Reimpresso por: New York: Kraus Reprint, 1971.

LENTINO, Noemia. Guia teórico, prático e comparado dos principais sistemas de classificação bibliográfica. São Paulo: Polígono, 1971.

LIBRARY OF CONGRESS. The Civil Rights History Project: Survey of Collections and Repositories - Howard University. Moorland-Spingarn Research Center. The American Folklife Center, Sept. 26, 2018. [Acesso em: 12 dez. 2020]. Disponível em: https://www.loc.gov/folklife/civilrights/survey/view_repository.php?rep_id=789.

LUPIN, Maurice A. Na important figure in Black Studies: Dr. Dorothy B. Porter. CLA Journal, v. 16, n. 4, p. 514-518, 1973. DOI: https://www.jstor.org/stable/44329017 
MADISON, Avril Johnson; WESLEY, Dorothy Porter. Dorothy Burnett Porter Wesley: Enterprising Steward of Black Culture. The Public Historian, v. 17, n. 1, p. 15-40, 1995. DOI: $10.2307 / 3378349$

MILLS, Jack. A morden outline of library classification. London, Chapman and Hall, 1960.

NUNES, Zita Cristina. Remembering the Howard University Librarian Who Decolonized the Way Books Were Catalogued. Smithsonian Magazine, 16 nov. 2018. [Acesso em: 11 nov. 2020]. Disponível em: https://www.smithsonianmag.com/history/remembering-howard-university-librarianwho-decolonized-way-books-were-catalogued-18097089o/

OMBUD. Why we named an engineering initiative after Dorothy Porter: the Dewey decimal de-colonizer, Ombud News, 2020. [Acesso em: 12 dez. 2020]. Disponível em: https://www.ombud.com/blog/ombud-engineering-initiative-dorothy-porter-deweydecimal-decolonizer.

OLSON, Hope. A potência do não percebido: Hegel, Dewey e seu lugar na corrente principal do pensamento classificatório. Tradução de Márcia Regina Silva. InCID: Revista de Ciência da Informação e Documentação, v. 2, n. 1, p. 3-15, jan./jun. 2011. DOI: https://doi.org/10.11606/issn.2178-2075.v2i1p3-15

OLSON, Hope. The Ubiquitous Hierarchy: An Army to Overcome the Threat of a Mob. Library Trends, v. 52, n.3, p. 604-616, 2004. [Acesso em: 9 set. 2021]. Disponível em: https://www.ideals.illinois.edu/handle/2142/1688

OLSON, Hope. The Power to Name: Representation in Library Catalogs. Signs, v. 26, n. 3, p. 639-668, 2001. [Acesso em: 10 dez. 2020]. Disponível em: https://www.jstor.org/stable/3175535

OLSON, Hope. Mapping beyond Dewey's boundaries: constructing classificatory space for marginalized knowledge domains. Library trends, v. 47, n. 2, p. 233-254, 1998. [Acesso em: 9 set. 2021]. Disponível em: https://www.ideals.illinois.edu/handle/2142/8210

PIEDADE, Maria Antonieta R. Introdução à teoria da classificação. 2. ed. Rio de Janeiro: Interciência, 1983.

PORTER, Dorothy B. HUNTON, Margaret R.; WILLIAMS, Ethel (ed.). A Catalogue of Books in the Moorland Foundation. Washington DC: Howard University, Compiled Under U.S. Works Progress Administration, 1939.

PORTER, Dorothy B. David Ruggles, na apostle of human rights. The Journal of Negro History. v. 28, n. 1, p. 23-50, 1943. [Acesso em: 10 dez. 2020]. Disponível em: https://www.journals.uchicago.edu/doi/10.2307/2714783.

PORTER, Dorothy B. Early American Negro Writings: A Bibliographical Study. Third Quarter, v. 39, n. 3, p. 192-268, 1945.

PORTER, Dorothy B. (ed.). A Catalogue of the African Collection in the Moorland Foundation Howard University Library. Washington, D.C.: Howard University Press, 1958. 
PORTER, Dorothy B. The African Collection at Howard University. African Studies Bulletin, v. 2, n. 1, p. 293-303, 1959. DOI: $10.2307 / 522962$

PORTER, Dorothy B. Documentation on the Afro-American: Familiar and Less Familiar Sources. African Studies Review, v. 12, n. 3, p. 293-303, dec. 1969. DOI: $10.1017 /$ So002020600037586

PORTER, Dorothy B. The Negro in the United States: a selected bibliography. Washington: Library of Congress, 1970.

PORTER, Dorothy B. Afro-Braziliana: a Working Bibliography. Boston: Beacon Press, 1978.

PORTER, Dorothy B. Interview. Em: SCARUPA, Harriet Jackson. The Energy-Charged Life of Dorothy Porter Wesley. New Directions, v. 17, n. 1, p. 1-12, 1990. [Acesso em: 13 dez. 2020]. Disponível em: http://dh.howard.edu/newdirections/vol17/iss1/3

PORTER, Dorothy B. Early Negro Writing 1760-1837. Baltimore: Black Classic Press, 1995.

PYNE, Charlynn Spencer. A wealth of African American Resources: Scholars' use of LC Colletction Inspires Seminar. Information Bulletin, Washington, 18 april 1994. [Acesso em: 13 dez. 2020]. Disponível em: https://loc.gov/loc/lcib/94/9408/seminar.html

SALDANHA, Gustavo S. Democracia documentária e a teoria da não-conceitualidade: filosofia e práxis. Informação \& Sociedade, v. 30, p. 21-41, 2020.

SALES, Rodrigo de. A Classificação de Livros de William Torrey Harris: influências de Bacon e Hegel nas classificações de biblioteca. Encontros Bibli: revista eletrônica de biblioteconomia e ciência da informação, v. 22, n.50, p.188-204, set./dez., 2017.

SCARUPA, Harriet Jackson. The Energy-Charged Life of Dorothy Porter Wesley. New Directions, v. 17, n. 1, p. 1-12, 1990. [Acesso em: 13 dez. 2020]. Disponível em: http://dh.howard.edu/newdirections/vol17/iss $1 / 3$

SIMS-WOOD, Janet. Dorothy Porter Wesley at Howard University: Building a Legacy of Blak History. Charleston: The History Press, 2014.

SILVA, Franciéle Carneiro Garcês da; SALDANHA, Gustavo Silva. Biblioteconomia Negra Brasileira: caminhos, lutas e transformação. Em: ENCONTRO NACIONAL DE PESQUISA EM CIÊNCIA DA INFORMAÇÃO, 20., 2019, Florianópolis. Anais...

Florianópolis: ANCIB; UFSC, 2019.

SILVA, Franciéle Carneiro Garcês da; SALDANHA, Gustavo Silva. As culturas africanas e afrodescendentes em Biblioteconomia \& Ciência da Informação no Brasil: Epistemologia histórica, pensamento crítico e meio social. Em: SPUDEIT, Daniella F. A. de O.; PEREIRA, Danielle B.; LOBÃO, Irajayna de S. L.; DAVID, Jéssica G. (org.). Formação e atuação política na Biblioteconomia. São Paulo: ABECIN Editora, 2018.

SILVA, Franciéle Carneiro Garcês da. Representações Sociais acerca das Culturas Africana e Afro-Brasileira na Educação em Biblioteconomia no Brasil. 2019. $521 \mathrm{f}$. Dissertação (Mestrado em Ciência da Informação) - Universidade Federal do Rio de Janeiro/Escola de Comunicação, Instituto Brasileiro de Informação em Ciência e 
Tecnologia, Programa de Pós-Graduação em Ciência da Informação, Rio de Janeiro, 2019.

SILVA, Franciéle Carneiro Garcês da. Colonialidade do saber e dependência epistêmica na Biblioteconomia: reflexões necessárias. Em: CARDONA, Natalia Duque; SILVA, Franciéle Carneiro Garcês da. Epistemologias Latino-Americanas na Biblioteconomia e Ciência da Informação: contribuições da Colômbia e do Brasil, Florianópolis: Rocha Gráfica e Editora, 2020. (Selo Nyota).

TENNIS, Joseph T. The Strange Case of Eugenics: A Subject's Ontogeny in a LongLived Classification Scheme and the Question of Collocative Integrity. Journal of the American Society for Information Science and Technology, v. 63, n. 7, p. 1350-1359, 2012. [Acesso em: 10 jan. 2021]. Disponível em: http://hdl.handle.net/1773/37943

TRAPP, Rafael Petry. Dorothy Porter e a constituição de um campo bibliográfico sobre o negro no Brasil e nos Estados Unidos (1943-1978). Topoi, Rio de Janeiro, v. 21, n. 45, set./dez. 2020. DOI: http://dx.doi.org/10.1590/2237-101x02104505

UZELAC, Costance P. Dorothy Porter Wesley (1905-1995). BlackPast, 2010. [Acesso em: 10 dez. 2020]. Disponível em: https://www.blackpast.org/african-americanhistory/dorothy-porter-wesley-1905-1995/

WEEKS, Linton. A luz undimmend. The Washington Post, Washington, 15 nov. 1995. [Acesso em 10 nov. 2020]. Disponível em: https://www.washingtonpost.com/archive/lifestyle/1995/11/15/the-undimmedlight/5e4330cc-4d2e-40d6-9c11-8af6e28b9c84/?utm_term=.92eo8cefb3c3

WIEGAND, Wayne A. Irrepressible reformer: a biography of Melvil Dewey. Chicago: American Library Association, 1996.

WIEGAND, Wayne A. The "Amherst Method": the origins of the Dewey Decimal Classification scheme. Libraries \& Culture, Austin, v. 33, 175-194, 1998.

ZALUDA, Scott. Lost Voices of the Harlem Renaissance: Writing Assigned at Howard University, 1919-31. College Composition and Communication, v. 50, n. 2, 1998, p. 232257. [Acesso em: jan. 2021]. Disponível em: www.jstor.org/stable/358515 